\title{
INFLUENCE OF ACCOMMODATION AND REFRACTIVE STATUS ON THE PERIPHERAL REFRACTIVE PROFILE
}

Leon Nicholas Davies ${ }^{1}$, Edward Arthur Harry Mallen²

${ }^{1}$ Ophthalmic Research Group, School of Life and Health Sciences, Aston University, Birmingham, B4 7ET, United Kingdom.

${ }^{2}$ Bradford School of Optometry and Vision Science, University of Bradford, Bradford, BD7 1DP, United Kingdom.

The Corresponding Author has the right to grant on behalf of all authors and does grant on behalf of all authors, an exclusive licence (or non-exclusive for government employees) on a worldwide basis to the BMJ Publishing Group Ltd and its Licensees to permit this article (if accepted) to be published in British Journal of Ophthalmology and any other BMJPGL products to exploit all subsidiary rights, as set out in our licence (http://bjo.bmj.com/ifora/ licence.pdf).

Keywords: accommodation; peripheral refraction; refractive error

Word count: 2448

\author{
Correspondance: \\ Leon N Davies, PhD \\ Ophthlmic Research Group \\ School of Life and Health Sciences \\ Aston University \\ Birmingham, B4 7ET \\ United Kingdom. \\ Tel: +44 (0) 1212044152 \\ Fax: +44 (0) 1212044048 \\ I.n.davies@aston.ac.uk
}




\section{Abstract}

Background: To determine, objectively and non-invasively, whether changes in accommodative demand modify differentially the peripheral refraction in emmetropic and myopic human eyes.

Methods: Forty subjects (19 male, 21 female) aged 20 to 30 years (mean \pm SD: $22.7 \pm 2.8$ years), twenty-one emmetropes (mean spherical equivalent refractive error $[\mathrm{MSE}] \pm$ SD: $-0.13 \pm$ $0.29 \mathrm{D})$ and 19 myopes (MSE \pm SD: $-2.95 \pm 1.76 \mathrm{D})$ participated in the study. Ametropia was corrected with soft contact lenses (etafilcon A, 58\% water content; Acuvue Dailies, Vistakon; Johnson \& Johnson Vision Care, Jacksonville, FL). Subjects viewed monocularly a stationary, high contrast (85\%) Maltese cross at $0.0,1.0,2.0$ and $3.0 \mathrm{D}$ of accommodative demand and at 0 , 10, 20 and 30 degrees field angle (nasal and temporal) through a +3.0 D Badal optical system. Static recordings of the accommodation response were obtained for each accommodative level, at each field angle, with an objective, open-view, infrared optometer (Shin-Nippon SRW-5000; Ryusyo Industrial Co. Ltd, Osaka, Japan).

Results: Peripheral mean spherical equivalent $(M)$ data showed that the emmetropic cohort exhibited relative myopic shifts into the periphery, while the myopic group showed hypermetropic shifts. Increasing accommodative demand did not alter the peripheral refractive profile in either the temporal $(p=0.25)$ or nasal $(p=0.07)$ periphery with no differential accommodative effect between refractive groups in either the temporal $(p=0.77)$ or nasal $(p=0.73)$ field. Significant shifts in the $J_{0}$ astigmatic component were seen in the temporal $(p<0.0005)$ and nasal $(p<$ 0.0005) fields with increasing eccentricity. Interaction effects between eccentricity and accommodative demand illustrated that increasing accommodative demand altered significantly the peripheral refractive profile in the temporal $J_{0}$ astigmatic component $(p<0.0005)$. The nasal periphery, however, failed to show such an effect $(p=0.65)$.

Conclusions: Alterations in peripheral refraction augmented by changes in ocular accommodation are relatively unaffected by refractive error for young, healthy human eyes. 


\section{Introduction}

It is established that both genetic [1] and environmental factors [2] have potential to play a role in the development of myopia. To date, the exact mechanism for the involvement of the visual environment in myopia development is uncertain, nevertheless evidence from animal studies suggests that retinal image quality may be an important factor.[3] Analogues may be drawn with human studies where periods of intense nearwork with a cognitive element have been shown to induce myopia in young adults, and re-trigger axial elongation in stable myopes.[4]

The role of hyperopic foveal blur, occurring during a near task as a result of accommodative lag, in the onset of myopia is equivocal.[5] Recent evidence, however, indicates that the correction of hyperopic defocus by the use of progressive addition spectacle lenses may attenuate the speed of myopia progression in children with a relatively high baseline lag of accommodation.[6] Of further interest is the potential role of peripheral retinal shape [7] and image quality [8] in refractive error development. Animal evidence has shown that form deprivation of the peripheral retina can lead to elongation of the vitreous chamber [9] and hence an increase in myopia. The degree of induced axial elongation appears to be related to the area of peripheral retina that is deprived of form, and recovery from experimentally induced refractive error appears possible without a clear foveal image.[9]

Data on the peripheral refractive status of the human eye have been reported previously, and have shown that the classical groups of central spherical refractive error are associated with specific retinal contours and patterns of peripheral refractive error.[10] In hyperopia, relative peripheral myopia is found due to a steepening retina; emmetropic eyes tend to show a spherical eye shape; myopic eyes exhibit relative hyperopia in the peripheral retina.[11] Further, oblique astigmatic effects induce an increase in astigmatism with greater field angles, with nasal-temporal asymmetry being observed.[12] 
Notwithstanding the plethora of literature dating back more than 75 years regarding peripheral refraction, few studies have examined directly the short-term influence of accommodation on peripheral refraction.[13, 14] Calver et al. [13] measured peripheral refraction at eccentricities up to 30 degrees at two levels of accommodative stimulation $(0.4 \mathrm{D}$ and $2.5 \mathrm{D})$, with targets in free space. No significant difference in peripheral refraction was seen between the distance target and the near target in either their emmetropic or myopic participants. Animal studies examining experimentally induced ametropias suggest that there are lenticular shape changes which may be linked with refractive error-associated global changes to the eye.[15] Moreover, Ronkina and coworkers suggested that the posterior capsule in myopic eyes is thicker than in emmetropic eyes.[16] Intuitively, one may suggest that these physiological lenticular differences between myopic and emmetropic eyes may give rise to disparate shifts in peripheral refractive status due to accommodative effort. Flitcroft's dioptric space model of the visual environment demonstrates graphically the range of accommodative stimuli to which the visual field can be exposed in a single scene.[17] Natural outdoor scenes tend to produce visual stimuli over a smaller dioptric range, compared to typical scenes from the workplace, where the dioptric range of stimuli to which the eye is exposed is considerably greater. As a consequence of this, the potential for localised defocus in the peripheral retina is considerable, which in turn can be modulated by retinal contour [18] and off-axis refractive error.[19]

As previous studies have shown differences in the accommodative response to targets conjugate with the fovea,[20] the hypothesis central to this study is that the modulation of peripheral retinal image quality by the action of accommodation, or more specifically the crystalline lens shape change during accommodation, may be different in emmetropic compared to myopic human eyes. Intuitively, such changes in peripheral retinal image quality as accommodation is manipulated may act at the level of the photoreceptors as a regulatory factor in eye growth. If such a system is disrupted by accommodative effort, it may predispose an individual to myopia. In this experimental work, the relative refractive status of the peripheral retina will be measured at 
different levels of accommodative effort. Any differences in peripheral refraction profile during the accommodation response between emmetropic and myopic individuals will be explored.

\section{Methods}

Forty subjects (19 male, 21 female) aged 20 to 30 years (mean \pm SD: $22.7 \pm 2.8$ years) were recruited. The cohort comprised twenty-one emmetropes (mean spherical equivalent refractive error $[\mathrm{MSE}] \pm$ SD: $-0.13 \pm 0.29 \mathrm{D}$, of which 15 were British Asian and 6 were White British) and 19 myopes (MSE \pm SD: $-2.95 \pm 1.76 \mathrm{D}$, of which 16 were British Asian and 3 were White British). Subjects in both refractive groups were gender matched (emmetropes: 10 men and 11 women; myopes: 9 men and 10 women). There was no significant difference in mean ages between refractive groups (emmetropes: $23.0 \pm 2.9$ years; myopes: $21.7 \pm 1.8$ years; unpaired $t$-test: $p=$ 0.10). To ensure that the accommodative demand was matched for each subject, all ametropia was corrected with soft contact lenses (etafilcon A, 58\% water content; Acuvue Dailies, Vistakon; Johnson \& Johnson Vision Care, Jacksonville, FL). All subjects had a visual acuity of 0.00 logMAR or better with an amplitude of accommodation $\geq 8.0 \mathrm{D}$, and uncorrected astigmatism was limited to $\leq 0.50 \mathrm{DC}$. A full ocular examination was performed on all subjects to rule out any ocular pathology, and any history of refractive surgery. Subjects were provided with a full explanation of the experimental protocol and gave written consent before commencing the investigation. All procedures were carried out in accordance with the tenets of the Declaration of Helsinki, and were approved by the Institutional Human Research Ethics Committee.

The experimental apparatus consisted of an objective, open-view, infrared optometer (ShinNippon SRW-5000; Ryusyo Industrial Co. Ltd, Osaka, Japan) attached to which was a +3.0 D Badal optical system with a stationary high contrast Maltese cross target (angular subtense: $10^{\circ}$; luminance: $37.0 \mathrm{~cd} / \mathrm{m}^{2} ; 85 \%$ Michelson contrast). The Badal system could be rotated to alter the fixation angle of the target relative to the centre of rotation of the subject's right eye (Figure 1). Subjects viewed monocularly the Maltese cross target at $0.0,1.0,2.0$ and $3.0 \mathrm{D}$ of accommodative demand and at 0,10,20 and 30 degrees field angle (nasal and temporal retina). 
Both the target distance and the field angle presentation order were randomised for each subject, using a set of shuffled cards. Five static recordings of the accommodation response were obtained and averaged for each accommodative level, at each field angle, with the SRW-5000 optometer.

Previous work has shown that peripheral refraction measurements remain valid with the addition of a soft contact lens.[21] To verify this, the refractive profile of a subset of 10 subjects (MSE: $1.80 \pm 0.91 \mathrm{D}$, range: -0.75 to $-3.50 \mathrm{D}$ ) was measured with and without contact lenses in situ.

Insert Figure 1 about here

Data analysis was performed on computer (Microsoft Excel; Microsoft Corp., Redmond, WA; SigmaPlot 2000, ver. 9.0; Systat Software UK Ltd, London, UK; SPSS for Windows, ver. 12; SPSS, Inc., Chicago, IL). The SRW-5000's sphere / cylinder / axis refractions (S/CX $\theta)$ were converted to vector components of spherical equivalent $(M), 90^{\circ}$ to $180^{\circ}$ astigmatism $\left(J_{0}\right)$, and $45^{\circ}$ to $135^{\circ}$ astigmatism $\left(J_{45}\right)$.

$$
\begin{gathered}
M=S+C / 2, \\
J_{0}=-C \cos (2 \theta) / 2, \\
J_{45}=-C \sin (2 \theta) / 2 .
\end{gathered}
$$

For illustrative purposes, data for each accommodative level in both refractive groups were fitted with a polynomial function. To find the best fit function, an iterative procedure was used where the order of the polynomial was increased systematically until the statistical power of the function was maximised. In all cases, the optimum fit was achieved with a quadratic function. In order to assess the statistical impact of accommodation on peripheral refraction and the anticipated interaction with refractive status, all raw data were treated with a 3-factor ANOVA where 
accommodative demand and eccentricity were taken as within-subject factors, and refractive grouping taken as a between-subject factor.

\section{Results}

At the fovea, the accommodative stimulus-response function was not significantly different between the emmetropes and myopes (Emmetropes: $y=0.67 x+0.32$; Myopes: $y=0.74 x+0.37$, $p=0.052)$. Peripheral mean spherical equivalent $(M)$ data showed that the emmetropic cohort exhibited relative myopic shifts into the periphery (up to approximately $1.0 \mathrm{D}$; Figure $2 \mathrm{a}$ ), while the myopic group showed hypermetropic shifts into the periphery (up to approximately 0.75 D; Figure $2 b)$. In both refractive groups, however, only the temporal retina exhibited a significant shift in $M$ with increasing eccentricity $\left(F_{(3,114)}=5.51, p=0.001\right)$, while the shift in the nasal periphery was not significant $\left(F_{(3,114)}=0.44, p=0.73\right)$. As expected, Figure 2 clearly demonstrates that the differences in profile between refractive groups was highly significant for both the temporal $\left(F_{(3,114)}\right.$ $=9.68, \mathrm{p}<0.0005)$ and nasal $\left(\mathrm{F}_{(3,114)}=13.11, \mathrm{p}<0.0005\right)$ periphery. The data for the interaction effects between eccentricity and accommodative demand, however, illustrated that increasing accommodative demand did not alter the peripheral refractive profile in either the temporal $\left(F_{(9,342)}\right.$ $=1.27, \mathrm{p}=0.25)$ or nasal $\left(\mathrm{F}_{(9,342)}=1.78, \mathrm{p}=0.07\right)$ periphery. Furthermore, there was no differential accommodative effect on the peripheral refractive profile between refractive groups in either the temporal $\left(F_{(9,342)}=0.63, p=0.77\right)$ or nasal $\left(F_{(9,342)}=0.67, p=0.73\right)$ field.

-Insert Figure 2 about here

Highly significant shifts in the $J_{0}$ astigmatic component were seen in the temporal $\left(F_{(3,114)}=\right.$ 516.66, $p<0.0005)$ and nasal $\left(F_{(3,114)}=260.94, p<0.0005\right)$ fields with increasing eccentricity. Figure 3, however, shows a level of asymmetry, with the temporal retina showing the greatest shift with increasing degrees of eccentricity; a phenomenon evident more so in the emmetropic cohort $\left(F_{(3,114)}=3.52, p<0.05\right)$. The results for the interaction effects between eccentricity and accommodative demand illustrated that increasing accommodative demand altered significantly 
the peripheral refractive profile in the temporal $J_{0}$ astigmatic component $\left(F_{(9,342)}=3.61, p<\right.$ 0.0005). The nasal periphery, however, failed to show such an effect $\left(F_{(9,342)}=0.76, p=0.65\right)$. Furthermore, there was no differential accommodative effect on the peripheral refractive profile between refractive groups in either the temporal $\left(F_{(9,342)}=1.32, p=0.22\right)$ or nasal $\left(F_{(9,342)}=1.21\right.$, $p=0.29)$ field

In contrast with both $M$ and $J_{0}$, the variation in the 45 to 135 astigmatism $\left(J_{45}\right)$ was very small across the visual field (Figure 4). Indeed, no single or combination of factors (angle, accommodation or refractive error) produced either a significant main effect or significant interaction.

Inclusion of a contact lens in a subset of myopic subjects induced a predictable shift in the overall refractive profile of the eye, compared to the non-lens situation (Figure 5). However, the shape of the peripheral refractive profile was not altered significantly by contact lens correction $\left(F_{(6,54)}=\right.$ $0.41, p=0.87)$. 


\section{Discussion}

In contrast to our hypothesis, we have shown that emmetropic and myopic subjects had similar peripheral refraction components whilst responding to a variety of accommodative targets in the horizontal visual field. Furthermore, the shapes of the best fit curves for $M, J_{0}$ and $J_{45}$ were comparable across the visual field showing no statistical or clinically relevant interaction between accommodative demand and refractive error. Our data add support to the findings of Calver et al. in a larger cohort and over a greater dioptric range of accommodative demands.[13]

As most visual stimuli in the modern environment are presented between optical infinity and 0.33 $\mathrm{m}$, we included accommodative demands which reflected this dynamic range (0 to $3 \mathrm{D})$. Despite this, and the range of refractive errors used (MSE: -6.00 to $0.50 \mathrm{D}$ ), both refractive groups showed similar shifts in peripheral refraction while accommodating to a variety of near targets. It is possible, however, that accommodative/refractive error changes in peripheral refraction might occur outside the refractive correction and accommodative range investigated in the study. Furthermore, alterations in the peripheral refractive profile may be present at more eccentric positions. Due to the ergonomics of the SRW-5000 autorefractor, however, this study was limited to measuring peripheral refraction out to an angle of $30^{\circ}$.

A variation from the lack of association between refractive error and peripheral refraction was the $J_{0}$ astigmatic component which showed a level of asymmetry. Here, the temporal retina showed the greatest change with increasing degrees of eccentricity. Indeed, this change in peripheral refraction was evident more so in the emmetropic cohort. This finding supports data from previous studies.[10, 11] The interaction effects between eccentricity and accommodative demand illustrated that increasing accommodative demand altered significantly the peripheral refractive profile in the temporal $J_{0}$ astigmatic component, while the nasal periphery failed to show such an effect. No differential accommodative effect on the peripheral refractive profile between refractive groups in either the temporal or nasal field was present. In accordance with previous 
investigations,[11, 22] however, our data also suggest that the eye's astigmatism is not at a minimum at the fovea, but rather in the nasal retinal periphery (Figure 3).

Studies of off-axis visual function have shown that spatial resolution of the peripheral retina is limited by mechanisms above the level of the photoreceptors rather than optical factors or photoreceptor density alone.[23] Therefore, correction of peripheral refractive error will not lead to a perceivable increase in the performance of parafoveal vision. However, animal studies have demonstrated that isolated areas of retinal blur can produce a localised compensatory change in eye shape,[24] and that axial elongation of the eye due to form deprivation is possible without the involvement of higher visual processing, for example in cases of optic nerve sectioning.[25] This experimental evidence supports the notion that structural recalibration of the eye, induced by the visual environment, is controlled at the level of the retina. However, our data suggests that peripheral refraction is modulated in a similar fashion by the accommodation response in both emmetropes and myopes. As a consequence, based on this evidence it would appear that changes in peripheral refraction associated with increased levels of accommodation, cannot be considered as a factor in myopigenesis. What remains unclear is the potential shift in higher order ocular aberrations away from the visual axis. Consequently, further study is required in this area. Our work is limited to a cross-sectional study of existing emmetropes and myopes. Further work to examine potential changes in the profile of peripheral refraction, and change in the modulation of peripheral refraction with accommodation, over a longitudinal period in emmetropes that remain emmetropic, and emmetropes that become myopic, may be of interest.

To conclude, we have shown that modifications in peripheral refraction augmented by changes in the level of ocular accommodation are relatively unaffected by refractive error for healthy human eyes. 


\section{Acknowledgements}

The authors would like to thank Saleel Jivraj for his assistance with subject recruitment and data collection and Richard Armstrong for his advice on statistical analysis. This work has been presented, in part, at the $11^{\text {th }}$ International Myopia Conference, Singapore (2006).

\section{Competing Interests}

The authors do not have any proprietary or financial interests in any of the devices or methods presented herein.

\section{References}

[1] Morgan I, Rose K. How genetic is school myopia? Prog Retin Eye Res. 2005 Jan;24(1):138.

[2] Saw SM. A synopsis of the prevalence rates and environmental risk factors for myopia. Clin Exp Optom. 2003 Sep;86(5):289-94.

[3] Wallman J, Winawer J. Homeostasis of eye growth and the question of myopia. Neuron. 2004;43:447-68.

[4] McBrien NA, Adams DW. A longitudinal investigation of adult-onset and adultprogression of myopia in an occupational group. Refractive and biometric findings. Invest Ophthalmol Vis Sci. 1997 Feb;38(2):321-33.

[5] Mutti DO, Mitchell GL, Hayes JR, Jones LA, Moeschberger ML, Cotter SA, et al. Accommodative lag before and after the onset of myopia. Invest Ophthalmol Vis Sci. 2006 Mar;47(3):837-46.

[6] Gwiazda JE, Hyman L, Norton TT, Hussein ME, Marsh-Tootle W, Manny R, et al. Accommodation and related risk factors associated with myopia progression and their interaction with treatment in COMET children. Invest Ophthalmol Vis Sci. 2004 Jul;45(7):2143-51.

[7] Logan NS, Gilmartin B, Wildsoet CF, Dunne MC. Posterior retinal contour in adult human anisomyopia. Invest Ophthalmol Vis Sci. 2004 Jul;45(7):2152-62. 
[8] Wang YZ, Thibos LN, Bradley A. Effects of refractive error on detection acuity and resolution acuity in peripheral vision. Invest Ophthalmol Vis Sci. 1997;38:2134-43.

[9] Smith EL, 3rd, Kee CS, Ramamirtham R, Qiao-Grider Y, Hung LF. Peripheral vision can influence eye growth and refractive development in infant monkeys. Invest Ophthalmol Vis Sci. 2005 Nov;46(11):3965-72.

[10] Millodot M. Effect of ametropia on peripheral refraction. Am J Optom Physiol Opt. 1981 Sep;58(9):691-5.

[11] Seidemann A, Schaeffel F, Guirao A, Lopez-Gil N, Artal P. Peripheral refractive errors in myopic, emmetropic, and hyperopic young subjects. J Opt Soc Am A Opt Image Sci Vis. 2002 Dec;19(12):2363-73.

[12] Dunne MC, Barnes DA. Modelling oblique astigmatism in eyes with known peripheral refraction and optical dimensions. Ophthalmic Physiol Opt. 1990 Jan;10(1):46-8.

[13] Calver R, Radhakrishnan H, Osuobeni E, O'Leary D. Peripheral refraction for distance and near vision in emmetropes and myopes. Ophthalmic Physiol Opt. 2007 Nov;27(6):584-93.

[14] Smith G, Millodot M, McBrien NA. The effect of accommodation on oblique astigmatism and field curvature of the human eye. Clin Exp Optom. 1988;71:119-25.

[15] Choh V, Sivak JG. Lenticular accommodation in relation to ametropia: the chick model. J Vis. 2005;5(3):165-76.

[16] Ronkina TI, Chabrova LS, Borisova LM, Vasin VI, Bagrova SN. The biomechanical properties of the crystalline lens capsule in emmetropia and myopia. Oftalmol Zh. 1989;7:420-5.

[17] Flitcroft DI. Dioptric space: extending the concepts of defocus to three dimensions. ARVO Annual Meeting. Fort Lauderdale, Florida, USA 2006.

[18] Atchison DA, Pritchard N, Schmid KL, Scott DH, Jones CE, Pope JM. Shape of the retinal surface in emmetropia and myopia. Invest Ophthalmol Vis Sci. 2005 Aug;46(8):2698-707.

[19] Charman WN, Jennings JA. Ametropia and peripheral refraction. Am J Optom Physiol Opt. 1982 Nov;59(11):922-3.

[20] Abbott ML, Schmid KL, Strang NC. Differences in the accommodation stimulus response curves of adult myopes and emmetropes. Ophthalmic Physiol Opt. 1998 Jan;18(1):13-20. 
[21] Artal P, Derrington AM, Colombo E. Refraction, aliasing, and the absence of motion reversals in peripheral vision. Vision Res. 1995 Apr;35(7):939-47.

[22] Dunne MC, Misson GP, White EK, Barnes DA. Peripheral astigmatic asymmetry and angle alpha. Ophthalmic Physiol Opt. 1993 Jul;13(3):303-5.

[23] Anderson SJ, Mullen KT, Hess RF. Human peripheral spatial resolution for achromatic and chromatic stimuli: limits imposed by optical and retinal factors. J Physiol. 1991 Oct;442:4764.

[24] Diether S, Schaeffel F. Local changes in eye growth induced by imposed local refractive error despite active accommodation. Vision Res. 1997 Mar;37(6):659-68.

[25] Troilo D, Gottlieb MD, Wallman J. Visual deprivation causes myopia in chicks with optic nerve section. Curr Eye Res. 1987 Aug;6(8):993-9. 


\section{Legends for Figures}

Figure 1 Schematic representation of the experimental apparatus, showing adjustments for field angle and accommodation stimulus.

Figure 2 Mean spherical equivalent refraction $(M)$ as a function of eccentricity $\left(-30^{\circ}\right.$ to $\left.30^{\circ}\right)$ for each level of accommodative demand (0 to $3 \mathrm{D}$ ) for (a) emmetropic and (b) myopic subjects. Error bars represent \pm 1 SEM. Note, some error bars are not visible where thay are similar to the size of the associated data points.

Figure 3 Mean $J_{0}$ astigmatism as a function of eccentricity $\left(-30^{\circ}\right.$ to $\left.30^{\circ}\right)$ for each level of accommodative demand ( 0 to 3 D) for (a) emmetropic and (b) myopic subjects. Error bars represent \pm 1 SEM. Note, some error bars are not visible where thay are similar to the size of the associated data points. Results for the $1 \mathrm{D}, 2 \mathrm{D}$ and $3 \mathrm{D}$ accommodative demands have been offset vertically for clarity by $-1 D,-2 D$ and $-3 D$, respectively.

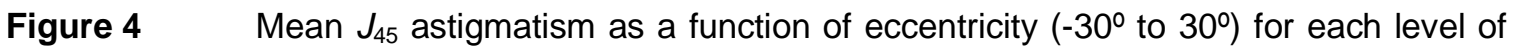
accommodative demand ( 0 to 3 D) for (a) emmetropic and (b) myopic subjects. Error bars represent \pm 1 SEM. Note, some error bars are not visible where thay are similar to the size of the associated data points. Results for the $1 \mathrm{D}, 2 \mathrm{D}$ and $3 \mathrm{D}$ accommodative demands have been offset vertically for clarity by $-1 \mathrm{D},-2 \mathrm{D}$ and $-3 \mathrm{D}$, respectively.

Figure 5 Mean spherical equivalent refraction $(M)$ as a function of eccentricity $\left(-30^{\circ}\right.$ to $\left.30^{\circ}\right)$ for a subset of myopic subjects $(n=10)$ with and without contact lenses in situ under distance fixation conditions. Error bars represent \pm 1 SEM. 
Field angle adustment $\left(+/-30^{\circ}\right)$

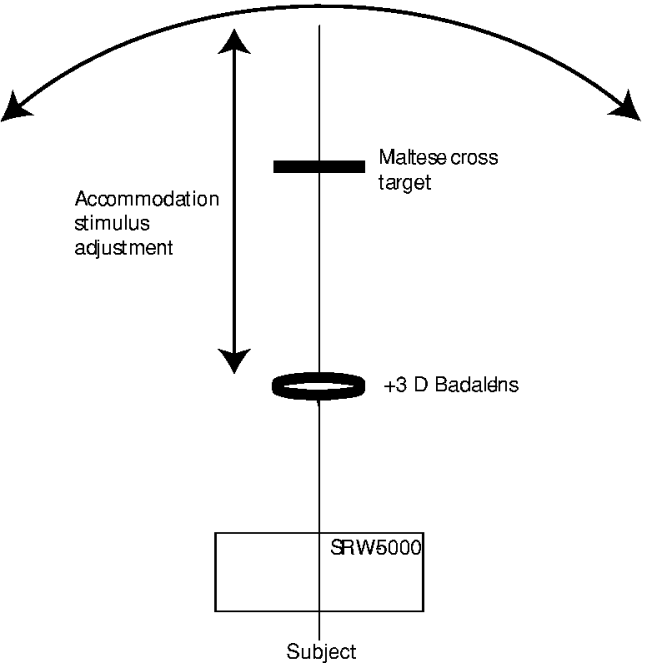



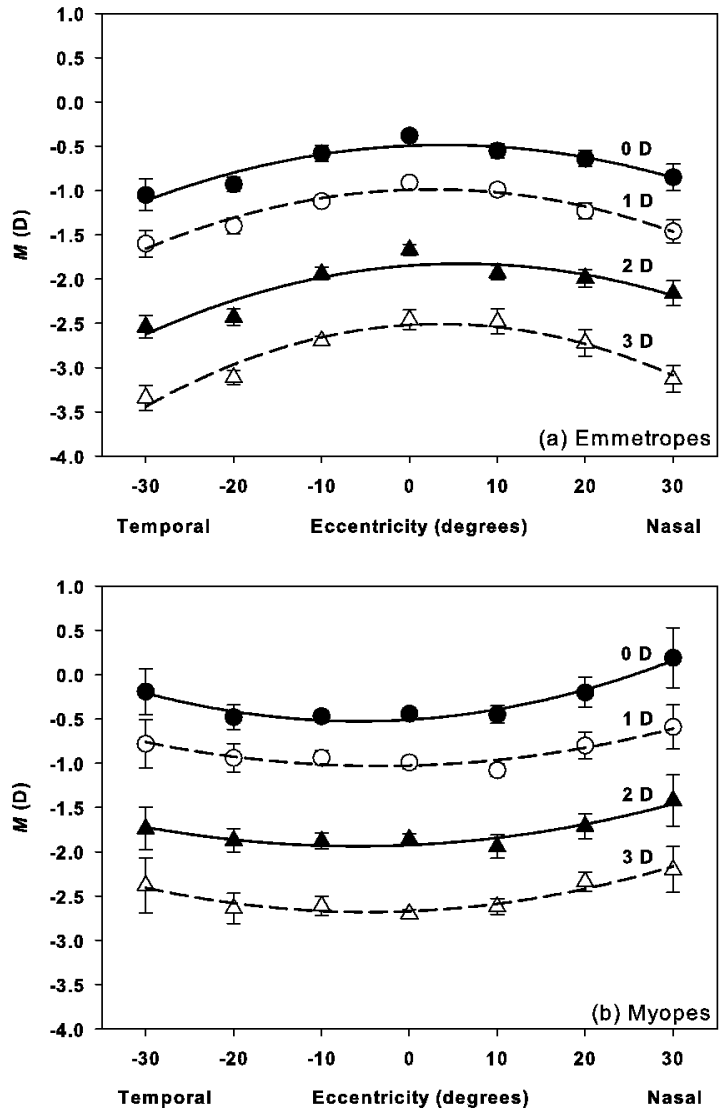

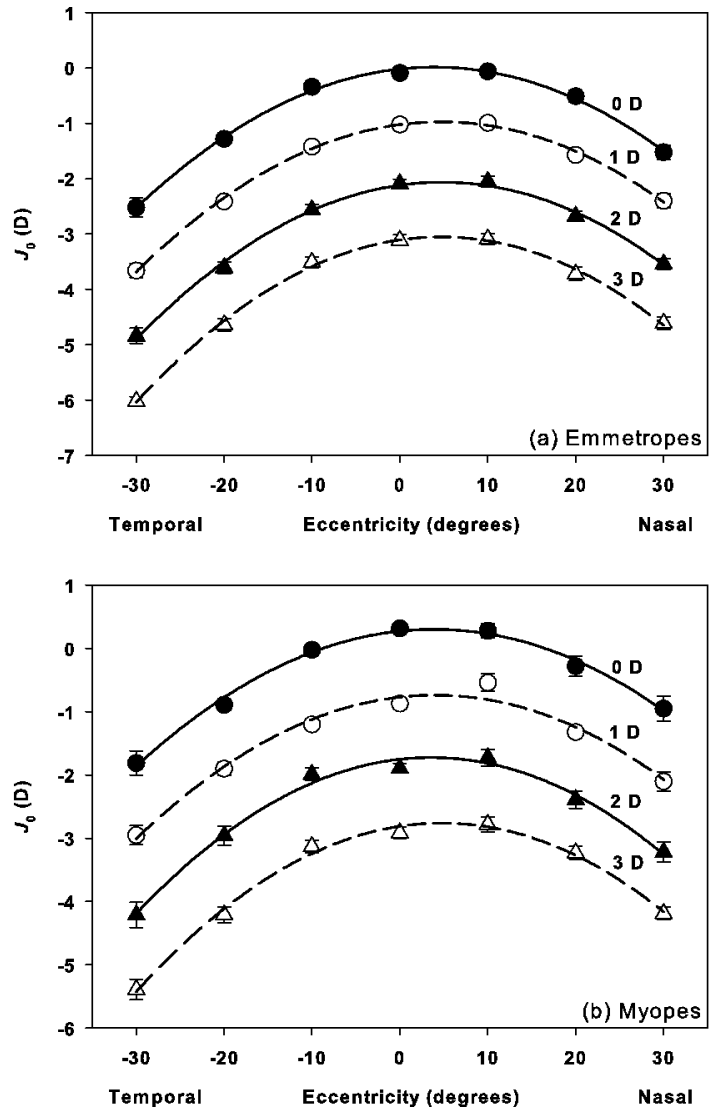


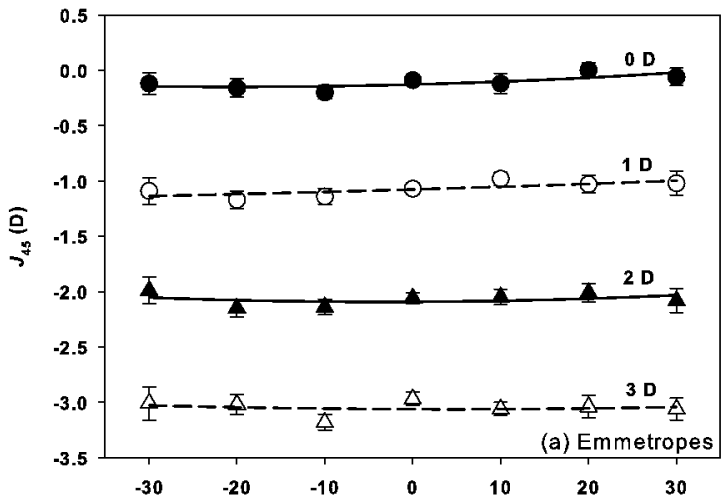

Temporal Eccentricity (degrees) Nasal

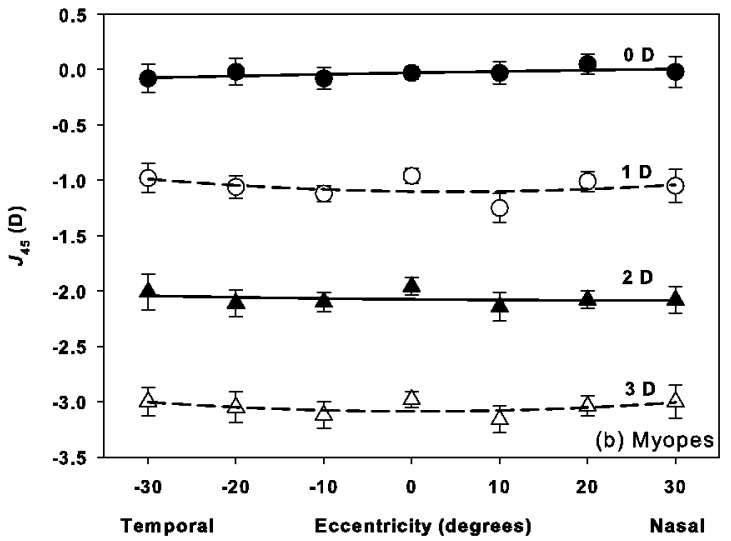




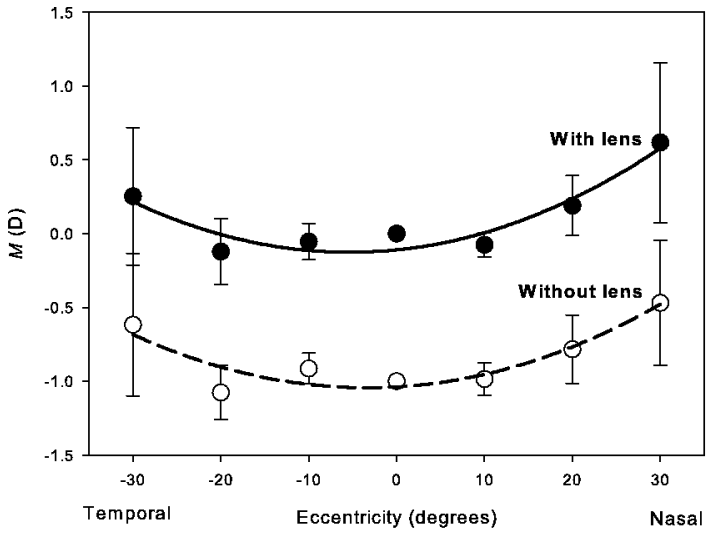

